\title{
THE RELATIONSHIP OF THE GONOCOCCAL COMPLEMENT FIXATION TEST TO THE DIAGNOSIS AND TREATMENT OF GONORRHEA
}

By I. N. ORPWOOD PRICE, D.P.H.

Mr. President, Ladies and Gentlemen,-During the last ten years the interest taken by clinicians and pathologists in the C.F.T. for gonorrhœa has greatly increased and by now most of you have had some experience of the results given by this test.

My object in giving this short paper is to give you a point of view (somewhat flavoured by the helpful, if occasionally harsh, criticisms of clinical colleagues) of a pathologist. I hope that when I have finished you will give the meeting the benefit of your experiences, so that we can take stock and bring up to date our opinions about what I believe to be a really valuable test.

Unfortunately, as in the case of the Wassermann reaction, there is no standard technique, but the report which I propose to submit is based on the results given by a technique which I elaborated in I930.

Most of you, I think, have little interest in the technical performance of the test, but there are three things I should like to bring to your notice.

(I) May I ask you to ensure that a reasonable quantity of blood is sent for examination. It is usually just as easy to take 8 c.c. of blood from a patient as I c.c., and an adequate amount of blood is of great help to the pathologist.

(2) Please send a few details about the patient and his complaint with each specimen to be examined. No honest pathologist cooks his results or allows his judgment to be swayed because he has this information; but it does help him to check his results, and if a disagreement with the clinical data occurs, he is able to repeat the test and thus give a definite decision. In addition, it is dull to turn out results like an automatic machine and not 


\section{BRITISH JOURNAL OF VENEREAL DISEASES}

have any idea of the clinical condition of the patient at the time of the test.

(3) May I appeal to the powers that be to set up a training school for serologists, where, amongst other things, would-be pathologists may be taught to do complement fixation tests. The results given by these tests have suffered more criticism than most other pathological procedures simply because of the bad performance of a good technique. This is due to the fact that many pathologists have picked up their knowledge from books and the stray remarks of their seniors instead of receiving proper systematic training.

Clinical Application.-In applying the results given by the complement fixation test for gonorrhoa to the clinical condition of the patient at the time of the test, it is essential to have some idea of the immunological factors at work.

It must be clearly understood that the test is a serum reaction which registers the presence or absence of specific gonococcal antibodies in the blood; the reaction itself does not signify the presence or otherwise of gonococci in the tissues. In general terms it may be stated that the production of antibodies is part of an infection, and that the amount of antibody so produced varies directly with the amount of toxin absorbed, together with the intensity of the reaction of the tissues against that toxin. The quantity of toxin absorbed probably depends on the amount of reaction produced by the infection and whether the infection is "closed " or "open." If the infection is "open," and if good drainage of the toxic products from the area of infection is established, little or no toxic absorption occurs and then the antibody response remains in abeyance. In a "closed" infection, where the toxic products are unable to drain efficiently, toxic absorption occurs and consequently antibodies appear in the blood stream. Exceptionally, cases occur in which, in spite of an apparently "closed" infection, the tissues do not seem to react to the presence of toxin and little antibody response is evoked.

In applying these facts to gonorrhoea, it must be remembered that this disease is primarily a "surface" disease and that the genital tract, owing to its anatomical peculiarities, affords numerous opportunities for such an infection to become " closed." 


\section{RELATIONSHIP OF THE GONOCOCCAL TEST}

Therefore it may be stated that a positive complement fixation reaction is indicative of the presence of gonococcal antibodies in the blood as a result of absorption of the toxic products from a gonococcal infection. It follows from this that such a patient must be regarded as infectious. There are two possible fallacies in this argument : (I) the presence of antibody due to the administration of vaccine; and (2) persistence of immunity after cure. These I will deal with later.

Conversely, a negative reaction indicates the absence of gonococcal antibodies in the blood-serum, but this does not exclude the presence of a gonococcal infection. Thus, whilst a negative reaction will be recorded in the absence of a gonococcal infection, under certain conditions a negative result may be recorded when gonococci are present in the tissues.

Now the production of gonococcal antibody depends on :-

(I) The age of the infection.

(2) The anatomical spread of the disease.

(3) The reaction of the tissues to the presence of gonococci.

(4) The stage of the infection at which treatment is instituted and the degree of therapeutic efficiency.

(I) The Age of the Infection.-In the average untreated cases of gonorrhœa a positive result may be expected in 27 per cent. of cases during the first week after exposure to infection, in the second week 46 per cent., in the third week 70 per cent., the fourth week 88 per cent., and the fifth and sixth weeks roo per cent. These percentages have been obtained from the results of tests on the sera of 545 patients, and should, therefore, give a fair estimate of what is to be expected. One fact emerges; and that is that the longer the disease is allowed to go unchecked the greater the probability of a positive result.

(2) The Anatomical Spread of the Disease.-This depends on the age of the infection, the local reaction of the tissues and the treatment, if any, adopted.

In the male, if the infection is limited to the anterior urethra a negative, or at most a weakly positive, reaction $( \pm)$ is the rule, unless some of the deeper structures such as Cowper's glands have become involved, in which case a positive reaction $(+)$ is the rule. When the disease has spread to the posterior urethra a strongly positive $(+ \pm)$ 


\section{BRITISH JOURNAL OF VENEREAL DISEASES}

or $(++)$ reaction quickly results. The rapidity and frequency with which a negative reaction changes to positive when the posterior urethra becomes involved is possibly due to the greater vascularity of the posterior urethra, and to the high percentage of infection of the seminal vesicles which occurs when the posterior urethra becomes involved. It seems probable that spread of infection to the posterior urethra is followed in many cases by almost simultaneous involvement of the seminal vesicles. This may lead to conditions of a "closed" infection, and the excellent blood supply to these organs facilitates absorption of toxic material with the consequent early appearance of antibody in the blood stream and the speedy production of a positive serum reaction.

In the female, a positive reaction is rarely obtained when the clinical and pathological evidence suggests that the infection is confined to the urethra. When, however, the infection spreads to the upper portion of the cervical canal, the uterus or the Fallopian tubes, a strongly positive reaction $(+ \pm)$ or $(++)$ is the rule. Gonococcal Bartholinitis usually results in a positive $(+)$ serum reaction.

(3) The Reaction of the Tissues. - It would appear that once absorption of toxin occurs, with its resulting formation of antibody, a positive complement fixation test may be expected in as short a time as three to four days. For example, I have produced in myself and in rabbits a positive $(+)$ reaction in the short space of four days by the daily injection of vaccine. By the tenth day a strongly positive $(++)$ reaction was obtained. The vaccine, I c.c. of which was equivalent to 360 millions of organisms, was injected subcutaneously into myself and intravenously into the rabbits daily for nine successive days in doses beginning at 0.25 c.c. and working up to 2 c.c. The positive reaction became negative six weeks after the cessation of the vaccine administration.

It was hoped that some evidence of the fate of gonococcal antibody might have been obtained from the records of $\mathrm{I} 6$ cases treated with a preparation sold as "gonococcal antitoxin." Unfortunately of a total of I6 patients, the results in I2 are of no significance, since, although the reaction was still positive after four months, gonococci were present in the secretions. No opinion can be expressed, therefore, as to whether the positive 


\section{RELATIONSHIP OF THE GONOCOCCAL TEST}

reactions are due to the persistence of the gonococcal infection or to the presence of antibody introduced by the injections of serum. In 4 of the I6 cases, however, the serum reaction became negative within eight weeks of the cessation of the administration of the gonococcal antibody.

(4) The Stage of the Infection at which Treatment is Instituted.-(a) Acute Gonorrhoea.-If adequate treatment is adopted during the first week after infection, the reaction may only become weakly positive $( \pm)$, or it may even remain negative throughout the course of the infection. These cases are often rapidly cured, and lack of antibody in the blood stream (as shown by the negative serum test) is probably due to the localisation of the disease to the anterior portion of the urethra in the male, or in the case of the female to the lower and " surface" tissues of the genitalia with no involvement of the deeper structures.

(b) Subacute Gonorrhcea.-If the case is not seen until three or four weeks after exposure to infection, a positive reaction is generally recorded. In the male, it will frequently be found that the prostate and seminal vesicles have become infected and that gonococci can be demonstrated in the vesicular fluid by smears and cultures. In the female, it is often difficult to obtain positive smears and cultures in these subacute cases even when gonococci are almost certainly present, as, for example, in gonococcal " contact" cases. But confirmation of the clinical findings may frequently be obtained by means of the complement fixation test for gonorrhœa. At the same time search for the organism, the presence of which is the essential diagnostic criterion, must proceed.

(c) Chronic Gonorrhoea.-One frequently sees male patients who have passed into the chronic stage of the disease because they defaulted when being treated in the earlier stages of the infection. They may have little in the way of signs or symptoms, and the problem arises as to whether they are free from the disease. One method of helping to decide this question is by means of the complement fixation test. If this is positive the patient should be regarded as infectious, and a diligent search should be made for the focal site. The seminal vesicles as well as the prostate are often involved. Digital examination of these organs may reveal clinical evidence 


\section{BRITISH JOURNAL OF VENEREAL DISEASES}

of pathological change, and gonococci may be found in the vesicular fluid by means of smears and cultures. Efficient treatment, such as digital massage, will promote drainage from such a focus of infection and the complement fixation test will usually become negative. It must be clearly realised that, if a positive serum reaction has become negative after a course of massage, this is no guarantee that all gonococci have been removed; and, in such a case, one negative serum result must not be taken as indicating that the patient is cured. It does, however, show that drainage has been established, and that absorption of toxin has ceased. If the treatment is prematurely stopped, the serum test may later again become positive. This shows that active gonococci still exist and that absorption of toxin has recommenced. This is of the greatest importance in considering the value of the complement fixation reaction as a test of cure. Serological tests run parallel with the efficacy of treatment up to the point where adequate drainage is established and absorption of toxin has ceased. Thereafter, the test will remain negative as long as efficient drainage is maintained or if the disease has been eradicated. If treatment has been stopped prematurely because of one negative serum test, then the complement fixation test will again become positive.

The complement fixation test is very frequently positive in cases of chronic gonorrhœa in the female, since the infection in these cases usually involves the uterine cavity and its adnexa, which from their anatomical arrangement tend to form a "closed" focus of infection. These organs are very vascular (drainage from the uterus is difficult to establish), and therefore toxic absorption is favoured. Successful treatment in these cases is indicated by a falling off of the strength of the serum reaction, but until a positive reaction is reduced to a negative the patient should be regarded as infectious. When a positive reaction becomes negative the patient is, as a general rule, cured; although a cervicitis due to other organisms, such as staphylococci or the colon bacillus, may still exist.

Complications of Gonorrhoea.-Serologically, gonococcal complications may be divided into two groups :-

(I) Acute complications usually supervene when the serum test is already positive, and usually result in it 


\section{RELATIONSHIP OF THE GONOCOCCAL TEST}

becoming strongly positive $(++)$. Thus, an acute epididymitis or salpingitis may cause so much toxic absorption and tissue reaction that a strongly positive complement fixation test is recorded even when the serum is diluted to $I / 80$ (neat serum is used in the standard test).

(2) Chronic complications such as a chronic gonococcal arthritis, tenosynovitis, or chronic gonococcal rheumatism usually record a positive $(+)$ reaction $(80-90$ per cent.).

The more frequent use of the complement fixation test for gonorrhœa would lead to a higher percentage of correct diagnoses of many " rheumatic " cases of doubtful origin.

\section{Gonococcal Vulvo-Vaginitis of Children}

In order to form an opinion about the value of the complement fixation test in the diagnosis of gonococcal vulvo-vaginitis I have collected some data from two groups of patients, whose serological tests have been performed in the laboratory of the Whitechapel Clinic.

The first group consists of 228 cases of gonococcal vulvo-vaginitis who were examined by Dr. Mascall at the Whitechapel Clinic. All the tests recorded were performed on sera taken from the patients at the time of their first attendance. Of these, 4I (I8 per cent.) were positive and $\mathrm{I} 87$ were negative. Of the $4 \mathrm{I}$ positives, 28 were confirmed by cultures and smears. The remaining I3 had no other evidence of infection until some time later, when positive smears and cultures were obtained in every case.

Dr. Nabarro has kindly supplied me with the figures of the second group of patients. These children, 65 in number, attended the Clinic at Great Ormond Street for vulvo-vaginitis. In 27 of these, no clinical or pathological evidence of a gonococcal infection was found and negative gonococcal complement fixation tests were obtained. The remaining 38 patients were diagnosed clinically and pathologically as suffering from gonococcal vulvovaginitis. Serum was taken from each patient for this test three weeks after the first attendance at the Clinic. In this way it was anticipated that a greater number of positive serum reactions would be obtained. This proved to be the case ; and of a total of 38 patients, 12 ( 32 per cent.) gave a positive serum reaction. Thus no false positive results were recorded in this group of patients. 


\section{BRITISH JOURNAL OF VENEREAL DISEASES}

Further work on this question of the value of the complement fixation test for gonorrhœa in the diagnosis of the disease is required, and Dr. Curtis, of the Hospital for Sick Children, Great Ormond Street, is at present carrying out an investigation which should in a few years yield valuable information.

One can, however, hazard the opinion that the test is a useful one in the diagnosis of this complaint and should be used as a routine test.

\section{Cross-Fixation}

The question of cross-fixation with the antibodies of allied organisms such as the Micrococcus catarrhalis, the meningococcus and the Micrococcus flavus (particularly type 2) is sometimes raised. It undoubtedly becomes of importance when a patient's serum gives a positive complement fixation test for gonorrhœa in the absence of clinical or other pathological evidence of this disease, whilst definite or indefinite catarrhal symptoms of the nose and throat may be present. The possibility of this phenomenon has often been discussed, but usually with inconclusive results.

In order to provide some data from which to draw conclusions the following experimental work was performed.

Antigens of the gonococcus, $M$. catarrhalis, the meningococcus and $M$. flavus were titrated separately against their respective antisera. The antisera were obtained by injecting rabbits and guinea-pigs with the respective organisms.

Complete fixation was obtained by gonococcal antiserum even when gonococcal antigen is used in a dilution of I/I30, whilst with a catarrhalis antiserum complete fixation occurred when the gonococcal antigen was diluted to $\mathrm{I} / 30$. The meningococcal antiserum gave complete fixation when the gonococcal antigen was diluted to $\mathrm{I} / \mathrm{I}_{5}$; whilst, if the latter were used at a strength of I/ Io only, incomplete fixation was given by the flavus antiserum. No fixation occurred with a normal serum at an antigen dilution of approximately I/I5, whilst the serum controls proved to be free from anti-complementary action. It should be noted that both the flavus and meningococcal antisera and antigens employed were of a polyvalent type. 


\section{RELATIONSHIP OF THE GONOCOCCAL TEST}

Since the titre of such a gonococcal antigen would be taken as I in 30 (i.e., half that amount of antigen which just failed to fix 3 m.h.d. of complement in the presence of a normal serum), it was concluded that in routine tests the possibility of cross-fixation with antibodies of the meningococcus or $M$. flavus could be ignored. It is evident, however, that a degree of cross-fixation with the antibodies of $M$. catarrhalis does occur under experimental conditions. In fact, when such an antiserum as that used in the above experiment is tested by the standard gonococcal complement fixation test, a weakly positive $( \pm)$ reaction is recorded.

Further experiments on similar lines were conducted by titrating serial dilutions of catarrhalis, meningococcal and flavus antigens against gonococcal, catarrhalis, meningococcal and flavus antisera respectively.

These experiments show that if the antigens are used at their respective titres as previously determined, the only possibility of a cross-fixation reaction occurring during the performance of the complement fixation test for gonorrhœa would be when a potent catarrhalis antiserum was tested.

\section{Clinical Evidence}

The evidence which I have been able to collect clinically. is meagre but instructive. Three types of cases are represented.

(i.) Catarrhalis Infection.-A female child (aged nine months) had suffered from snuffles since birth, and as the mother had been treated for gonorrhœa one year previously, it was thought (as the Wassermann reaction was negative) that the child's complaint might be due to a nasal gonococcal infection. Gram-negative diplococci were found in the nasal discharge. Morphologically and culturally these were identical with Micrococcus catarrhalis. In the meantime the child's serum, which had been tested by the complement deviation reaction, recorded a negative result with a gonococcal antigen, and a strongly positive $(++)$ result with a catarrhalis antigen. Thus the causal organism was demonstrated serologically with the complete absence of any cross-fixation.

(ii.) Catarrhalis Infection.-A male child aged fifteen months had suffered from a profuse nasal discharge for 


\section{BRITISH JOURNAL OF VENEREAL DISEASES}

one year. The mother was being treated for a vaginal discharge at another hospital, and the child was sent for investigation in view of the fact that its blood serum had given a positive C.F.T. $(+)$ for gonorrhœa. This was repeated and found to be positive $(+)$ again. In the meantime nose and throat cultures had yielded Gramnegative diplococci which on investigation were morphologically and culturally $M$. catarrhalis. A complement deviation test for $M$. catarrhalis was then performed and gave a strongly positive reaction.

(iii.) Meningococcal Infection.-Through the courtesy of Dr. Coyle, who supplied me with specimens, I was able to examine the blood and cerebrospinal fluid from six children who were suffering from cerebrospinal meningitis. In each case the duration of the disease was a week or more, and they had all been treated with antimeningococcal serum. The blood sera of all six cases were negative when tested by the complement fixation test for gonorrhœa. The same test when applied to the cerebrospinal fluids gave five negative results, whilst the sixth had a weakly positive $( \pm)$ reaction. Unfortunately, at this time a meningococcal antigen was not available, and so I was unable to test these specimens by a meningococcal complement fixation reaction. This would have afforded an interesting comparison, but even so, it would appear from the results obtained that cross-fixation with antibodies of the meningococcus during a meningococcal infection is very unlikely to occur.

We may therefore conclude that the probability of cross-fixation reactions occurring with the antibodies of other Gram-negative cocci is excluded, with the possible exception of the Micrococcus catarrhalis. Such a crossfixation reaction with the antibodies of $M$. catarrhalis appears to be rare and, coupled with the weakness of this reaction, it is very doubtful if the result of such a test would confuse the diagnosis. Should any doubt arise, it can be dealt with by performing parallel complement fixation tests using gonococcal and $M$. catarrhalis antigens and comparing the relative strengths of their reactions.

At this stage I should like to point out that the test can be used as a means of identifying the gonococcus. If a pure culture of a Gram-negative diplococcus requires identification apart from the sugar reaction tests, it should be made up as a standard gonococcal antigen and 
titrated against a normal serum as well as a known strongly positive gonococcal antiserum. If no fixation of complement occurs in the presence of a normal serum when the antigen is diluted $\mathrm{I} / 20$ or more, and the complement is completely fixed in the presence of a known strongly positive gonococcal antiserum, when the antigen is diluted I/IOo or more, it is strong presumptive evidence that the organism used in making the antigen was a gonococcus.

Vaccines.-The effect of vaccines on the strength of the complement fixation reaction largely depends on the type of vaccine used as well as on the tissue reaction of each particular patient. It is frequently stated that the administration of vaccine may nullify the value of the complement fixation test for gonorrhœa. After careful study and experiment I have come to the conclusion that this is not so. As a result of injecting vaccine one of two things may happen. Either :-

(i.) The vaccine may have no effect and may fail completely to stir up the infection (in this case the strength of the serum reaction may show a temporary rise, and in my experience this returns to its original value within six to eight weeks after the last injection) ; or

(ii.) The vaccine may cause a "flare-up" at the focus of infection and in effect cause a chronic manifestation of the disease to become acute. The result serologically is usually a strongly positive $(++)$ reaction, and whilst this may be due in part to the reaction of the tissues against the vaccine, the clinical response suggests absorption of toxin elaborated by gonococci which have been stimulated into action by the vaccine. As stated above, the vaccine reaction ceases to show serologically about six to eight weeks after the last injection. So that if, after this period of time, the strength of the complement fixation test remains at a higher level than it was before the administration of vaccine, in all probability this is due to the absorption of toxin of the causative organs of the disease. Therefore if vaccine has been given during the course of the disease, any subsequent positive serum reaction is not of necessity due to the vaccine.

Sulphanilamide.-Sulphanilamide and its allied compounds are being extensively used in the treatment of gonorrhœa, and in order to ascertain what type of serum reaction might be expected, I have investigated $5 \mathrm{I}$ male 


\section{BRITISH JOURNAL OF VENEREAL DISEASES}

patients who have been treated by these compounds. All these patients had two things in common when the treatment was commenced : (I) a negative complement fixation test for gonorrhœa; (2) gonococci were present in the urethral smears. They are divided into two groups : (I) 28 patients treated with Prontosil album; (2) 23 patients treated with uleron.

Of the 28 patients treated with Prontosil album, II were not given any irrigations and the sera of 8 of these recorded positive reactions, whilst 3 remained negative throughout. Of the remaining I7 who were given irrigations, the sera of 7 recorded positive reactions, whilst Io remained negative throughout.

Of the 23 patients treated with uleron, 6 were not given any irrigations and the sera of 5 gave positive reactions, whilst I remained negative throughout. Of the remaining I7 who were given irrigations, the sera of ro became positive, whilst 7 remained negative throughout the course of the disease.

\begin{tabular}{l|c|c|c|c}
\hline & & Positive. & Negative. & Total. \\
\hline \multirow{3}{*}{ Prontosil } & Without irrigation & 8 & 3 & II \\
\cline { 2 - 5 } & With irrigation & 7 & Io & I7 \\
\hline \multirow{3}{*}{ Uleron } & Without irrigation & 5 & I & 6 \\
\cline { 2 - 5 } & With irrigation & Io & 7 & $\frac{\text { I7 }}{5 \text { I }}$ \\
\hline
\end{tabular}

Although the number of patients investigated is small, it would appear that :-

(I) Prontosil album is somewhat more effective than uleron in preventing the occurrence of a positive serum reaction.

(2) If treatment by either of these compounds is accompanied by irrigations, fewer positive serum reactions appear to be recorded.

\section{INTERPRETATION OF RESULTS}

It must be realised that this test is no more than an accessory method of diagnosis to be interpreted by the 


\section{RELATIONSHIP OF THE GONOCOCCAL TEST}

clinician in the light of the clinical findings. At the same time, it is often useful to have some idea of the "serological course" of the disease. The following scheme for a sequence of serological tests during the course of an infection is suggested for use in V.D. clinics. The patient's serum should be tested on the first appearance at the clinic. Whether the result of this be negative or positive, it should be repeated a month later, and thereafter at monthly intervals until such time as clinically a test of cure is indicated. Further tests depend on all the evidence available : clinical, pathological and serological. If a series of tests be done, a curve of the serum reactions may be plotted which gives a graphic picture of the serological course of the disease and indicates to a large extent the effect of the treatment adopted.

The notation used is as follows :-

$$
\begin{aligned}
& ++ \text { very strongly positive } \\
& + \pm \text { strongly positive } \\
& + \text { positive } \\
& \pm \text { weakly positive } \\
& \text { - negative }
\end{aligned}
$$

In the absence of vaccine treatment :-

(I) A strongly positive result ( ++ or + \pm ) is reliable evidence that the patient is suffering from gonorrhœea and must be regarded as infectious. With such a result it may be taken as probable that in the male the posterior urethra and its communicating glands are infected and that in the female the infection has extended to the cervical canal and uterine adnexa. Such a result indicates that the infection is active and that toxic products are being absorbed into the blood stream in relatively large quantities. Acute gonococcal complications are very commonly accompanied by a strongly positive serum test.

(2) A positive reaction $(+)$ is definite evidence that the patient is suffering from gonorrhœa; this may occur at any stage of the disease. It is usually an indication that the posterior urethra in the male, or the upper part of the cervical canal in the female, is infected.

(3) A weakly positive reaction ( \pm ) is more difficult to interpret, and such a result should be accepted with some reserve. Nevertheless, when successive specimens of sera from a particular patient give similar results a careful search will often reveal a focus of gonococcal infection. 


\section{BRITISH JOURNAL OF VENEREAL DISEASES}

At the same time a weakly positive reaction is likely to be obtained in the following circumstances:

(i.) An early case at the stage of one to three weeks after exposure to infection ;

(ii.) With acute urethritis when the infection is limited to the anterior urethra ;

(iii.) Old-standing cases in which, owing to effective treatment, the case is approaching final cure ;

(iv.) At any stage of the infection when the immunity processes are deficient.

(4) A negative reaction (-) obtained when gonococci are found in the secretions is evidence of :

(i.) An early acute infection of less than twenty-one days' duration ;

(ii.) An infection which, in the male, is limited to the anterior urethra, or, in the female, to the lower part of the genital tract.

(iii.) Infection of the posterior urethra in the male with adequate drainage from which the absorption of toxic material has ceased.

(5) A negative reaction (-) obtained when no gonococci are demonstrable in smears or cultures is confirmatory evidence that :-

(i.) The case is not a gonococcal infection; or

(ii.) The case is cured.

At this stage the value of the reaction in a test of cure may be discussed. It will be realised that a single negative serum test by itself is insufficient evidence on which to presume cure. A negative result shows that absorption of toxin has ceased, but this, although of great importance, is not conclusive evidence that the gonococci have been expelled from the tissues. Throughout a test of cure the following points should be remembered :-

(i.) It is likely that a patient with a persistently positive complement fixation test is still infectious. It is therefore useless to perform a test of cure until the complement fixation test for gonorrhœa has become negative.

(ii.) No patient should be discharged as cured on the strength of a single negative serum test, even when there is no clinical, microscopic or cultural evidence of infection.

(iii.) If a patient is prematurely discharged as cured on the strength of a single negative complement fixation test 


\section{RELATIONSHIP OF THE GONOCOCCAL TEST}

and gonococci are still present in the tissues, the serum test will often become positive again after an interval of about one month.

To sum up, in view of what has been already stated, it will be seen that in order to interpret correctly the result of a complement fixation test for gonorrhœa, it is essential to understand the significance of an " open " or " closed " infection. Either type of infection (or a modification thereof) may be present at any stage of the disease, and it may be surmised from the result of a serum reaction whether or not drainage is established. Thus a strongly positive reaction suggests a "closed" infection with little or no drainage and considerable absorption of the toxins of the infection. On the other hand, a negative result in a known case of gonorrhœa is usually evidence of excellent drainage and little or no absorption of toxin. Between these two extremes varying stages of drainage and absorption of the toxic material occur, and a varying serum reaction at any time during the disease is dependent on the extent to which these factors operate at the time of the test. If these facts be borne in mind, there will be little difficulty in correlating the result of a serum test with the clinical picture. Two exceptions to the above general rule occur :-

(i.) Patients (O.I per cent.) who appear to lack the necessary tissue reaction to form antibodies to the gonococcus ; and

(ii.) Patients who have been treated with vaccine.

A negative complement fixation test, therefore, does not of necessity exclude a gonococcal infection, but must be interpreted in the light of the clinical condition of the patient, together with any other pathological findings (smears, cultures) which are available.

On the other hand, providing no vaccine has been administered, a positive test is almost always indicative of the presence of living gonococci in the tissues. Some authorities have stated that a positive complement fixation test persists for more than two months after the patient has been cured. From the results of experiments with vaccine and from observation of cases, I do not believe that this is true. I doubt if gonococcal antibody remains in the blood stream for more than six to eight weeks unless a focus of active gonococci is present. If a positive serum reaction is obtained when the patient is 
apparently cured, further careful search will, as a rule, reveal a focus of infection.

Early acute untreated cases of gonorrhœa may be expected to give a positive serum test at any time from the first week onwards after exposure to infection.

Chronic cases of gonorrhœa which have been insufficiently treated frequently give a positive result.

Patients with chronic gonorrhoea who have been adequately treated so that efficient drainage is established may give a negative result; but if in such a case treatment is prematurely stopped on the evidence of one negative complement fixation test, a positive result is often obtained after a further interval of one month.

The test is an essential part of the investigation to establish cure; but if a negative result is recorded it should be confirmed by repetition as well as by smears and cultures. It cannot be too strongly emphasised that no patient should be discharged on the evidence of a single negative complement fixation test.

The test should be used to estimate the efficiency of antibody formation as a result of vaccine treatment.

Finally, there is little evidence to show that crossfixation with the antibodies of other Gram-negative cocci would confuse the issue either in diagnosis or in a test of cure. 